\title{
DISTRIBUTION OF MOBILE GENETIC ELEMENTS IN CANCER GENES
}

\author{
Preeti P. ${ }^{1}$, Tapan Kumar Baral ${ }^{1}$, Kamal Rawal ${ }^{1}$
}

1. Amity Institute of Biotechnology, Amity University Uttar Pradesh, India.

Corresponding author

Dr. Kamal - Centre for Computational Biology and Bioinformatics, Amity Institute of Biotechnology, Amity University, Noida, Uttar Pradesh, India

Email - kamal.rawal@gmail.com

\begin{abstract}
Mobile genetic elements (MGEs) represent a large portion of the human genome. Its ability to change their position within the genome has contributed to evolution, however, the same has also resulted in several mutations. Many of such mutations are known to cause exon skipping or premature truncation that result in non-functional or dysfunctional protein, leading to cancer. Here, in this study we aim to determine the distribution of MGEs in cancer-associated genes as compared to non-cancer associated genes. We curated a list of genes for both the categories and downloaded the nucleotide sequences of these genes and ran on RepeatMasker to identify the MGEs in each gene. All the data generated with respect to each gene was parsed and compared. The results showed that the number and the sequence length covered by the identified MGEs in cancerassociated genes were comparatively high. The abundance of MGEs may be correlated with the high risk of deletion/insertion of large DNA segments in these genes, that results in higher risk of cancer. Further studies need to be performed for better clarity on these associations.
\end{abstract}

Keywords: Mobile genetic elements, RepeatMasker, Oncogene, Proto-oncogene, Cancer

\section{INTRODUCTION}

MGEs are the DNA segments that can change their location within the genome. They are present in the genomes of most of organisms which includes, prokaryotes as well as eukaryotes. In humans, they occupy large fraction ( $>50 \%$ ) of the genome [Cao et al. 2020]. MGEs can be broadly classified into retrotransposons class (Class I) and DNA transposons (Class II), based on their mode of transmission. Out of two classes, retrotransposon variety is the most widely spread MGEs in mammals. Retrotransposons can be further categorized into Long Terminal Repeats (LTRs) and non-LTRs [Anwar et al. 2017]. Autonomous non-long terminal repeat retrotransposons are commonly referred to as long interspersed elements (LINEs). Short non-autonomous elements that borrow the LINE machinery are called SINES [Bakre et al 2005]. 
Though, majority of the MGEs are inactive in human genome, there are varieties of non-LTRs (like LINEs and SINEs) that are capable of generating mutations. These active MGEs has potential to disrupt genes and play role in chromosomal rearrangements [Anwar et al. 2017]. MGEgenerated mutations have shown to be associated with diseases affecting humans such as Mankes syndrome, X-linked agammaglobulinemia, Dent disease, hemophilia, cholinesterase deficiency, hereditary nonpolyposis colorectal cancer, familial adenomatous polyposis, leukemia, and cystic fibrosis [Hancks et al. 2012]. In a study performed by Wimmer et al. (2011), on neurofibromatosis type 1 patients, 18 unrelated patients were identified with MGE insertions in NF1 gene in exonic or intronic region. Studies have also shown that the insertion of mobile DNA in genes might promote exon skipping, resulting in either reduced or no expression of the affected gene(s) [Payer et al. 2019; Nikopoulos et al. 2019; Jourdy et al. 2018]. In 1988, Kazazian et al. discovered that TE insertion was responsible for causation of hemophilia A. Large-scale genomic projects, such as the ENCyclopedia Of DNA Elements (ENCODE) and Functional Annotation of Mouse (FANTOM) projects as well as independent studies have indicated that TEs are active in a highly cell type-specific manner and control their own cell-specific transcription as well as the transcription of neighboring genes [Faulkner et al. 2009; Djebali et al. 2012; Thurman et al. 2012]. Previously, our group identified unique patterns/signals at the insertion sites flanking MGEs using machine learning [Rawal and Ramaswamy 2011]. In this work, we found that signals present in exonic regions are different from the intronic regions of the genes. All these studies indicate the importance of understanding the role of MGEs insertions in human genes. Here, in this study we aim to elucidate the distribution of MGEs on oncogenes (proto-oncogene) and also to compare it with the non-cancer genes.

\section{MATERIAL AND METHOD}

Datasets:

We extracted a list of proto-oncogene or oncogenes from open source databases, including OMIM (https://omim.org/), Genetics-MedlinePlus (https://medlineplus.gov/genetics/gene/), and Cancer

Genetics Web (http://www.cancerindex.org/geneweb/) [labelled as Set T]. We also downloaded a whole list of human genes from NCBI database (https://www.ncbi.nlm.nih.gov/gene/?term=human[organism]) [Set W]. Next, we subtracted Set $\mathrm{W}$ by removing the Set $\mathrm{T}$ genes, as well as removed genes without RefSeq ID. The filtered list contains non cancer genes [Set $\mathrm{N}]$ as control dataset.

Nucleotide Sequence Download

We extracted the nucleotide sequences of genes in Set $\mathrm{T}$ and $\mathrm{N}$ using Edirect utilities. We divided Set N into 5 different sub-sets, N1, N2, N3, N4 and N5. Each subset consist of randomly selected 1082 genes as control dataset.

\section{MGE Detection}

We used RepeatMasker to identify and characterize the MGEs on gene sequences belonging to different set. Next, we used in-house scripts (Bash) that parsed RepeatMasker output files. 


\section{Statistical Analysis}

We computed standard statistical measures such as mean and standard deviations for the data obtained from Repeatmasker. Next, we compared these values in different sets (namely $\mathrm{T}, \mathrm{W}$ and $\mathrm{N})$ using T-test $(P$ values $<0.05)$.

\section{RESULTS}

Data Collection:

We identified 1164 genes in Set $\mathrm{T}$ that are reported to be associated with cancer or tumor (See Supplementary Table 1). We also downloaded a list of 61746 human genes in Set W (See Supplementary Table 1). We filtered Set W for all the matches with tumor/cancer keywords. We also filtered genes having no information on RefSeq ID leading to a total of 9994 genes in Set N (See Supplementary Table 1).

Nucleotide sequence download

From Set T, we could extract the nucleotide sequences of 1082 genes out of 1164 . This was because of the unavailability of the nucleotide sequence for few genes or the alias of gene name might be used in Edirect to refer to the gene (See File 4). Similarly, for Set N, we could download 9454 sequences out of 9994 genes present in the list.

\section{MGE Detection}

All the extracted nucleotide sequences were run on RepeatMasker to identify the MGE as well as other details such as coordinates, type, score etc. (see Table 1). We found that that number of MGEs in Set T (cancer genes) were higher as compared to Set N (control) (see Figure 1). We also compared the percentage of sequence covered by MGEs and the sequence length covered by MGEs in both the sets (see Figure 2 and Figure 3).

Since the number of genes in Set N (control dataset) were more than that of genes of Set T, we constructed 5 subsets of Set N, each with 1082 genes. During the analysis, we found that the frequency of MGEs in Set T was higher as compared to all the other subsets of Set N (see Table 2).

\section{CONCLUSION AND FUTURE PERSPECTIVE}

In this study, we performed a comparison between the distribution of MGEs in genes that are associated with cancer and genes that are not associated with cancer. The results have shown the abundance of MGEs in cancer-associated genes. Though the reasons for this abundance is not known but it can be hypothesized that the presence of these MGEs can make these genes amenable for large deletions or duplications/insertions that could lead to pathogenic mutations in the gene, resulting in cancer. Further studies are needed to explore the role of MGEs in genes expression and cancer genomics. 


\section{Contribution of Authors}

This study was conducted in the overall guidance of KR, who contributed in protocol, critical evaluation of data and manuscript. Data was generated by TKB. Data analysis, cross-checking and manuscript writing was done by PP. All the statistical analysis was done by PP, TKM and KR. All the authors are responsible for the content of the manuscript.

\section{Acknowledgement}

We extend our sincere gratitude to Amity University for providing administrative and technical support required in the conduct of this study.

\section{Financial Support and Sponsorship}

Dr. Kamal Rawal acknowledges the support provided by DBT, Government of India for the grant of Department of Biotechnology, Ministry of Science and Technology, Government of India (Grant Id: BT/PRI7252/BID/7/708/2016). We also acknowledge grant from SERB, Department of Science Technology (Grant ID: CVD/2020/000842). Preeti was provided fellowship from the DBT grant. Tapan worked as volunteer in the project.

\section{REFERENCES}

1. Cao X, Zhang Y, Payer LM, et al. Polymorphic mobile element insertions contribute to gene expression and alternative splicing in human tissues. Genome Biol. 2020;21(1):185. Published 2020 Jul 27. doi:10.1186/s13059-020-02101-4.

2. Anwar SL, Wulaningsih W, Lehmann U. Transposable Elements in Human Cancer: Causes and Consequences of Deregulation. Int J Mol Sci. 2017;18(5):974. Published 2017 May 4. doi:10.3390/ijms18050974

3. Hancks DC, Kazazian HH Jr. Active human retrotransposons: variation and disease. Curr Opin Genet Dev. 2012;22(3):191-203. doi:10.1016/j.gde.2012.02.006

4. Wimmer K, Callens T, Wernstedt A, Messiaen L. The NF1 gene contains hotspots for L1 endonuclease-dependent de novo insertion. PLoS Genet. 2011;7(11):e1002371. doi:10.1371/journal.pgen.1002371

5. Payer LM, Steranka JP, Ardeljan D, et al. Alu insertion variants alter mRNA splicing. Nucleic Acids Res. 2019;47(1):421-431. doi:10.1093/nar/gky1086

6. Nikopoulos K, Cisarova K, Quinodoz M, et al. A frequent variant in the Japanese population determines quasi-Mendelian inheritance of rare retinal ciliopathy. Nat Commun. 2019;10(1):2884. Published 2019 Jun 28. doi:10.1038/s41467-019-10746-4

7. Jourdy Y, Janin A, Fretigny M, et al. Reccurrent F8 Intronic Deletion Found in Mild Hemophilia A Causes Alu Exonization. Am J Hum Genet. 2018;102(2):199-206. doi:10.1016/j.ajhg.2017.12.010 
8. Faulkner GJ, Kimura Y, Daub CO, Wani S, Plessy C, Irvine KM, Schroder K, Cloonan N, Steptoe AL, Lassmann T, Waki K, Hornig N, Arakawa T, Takahashi H, Kawai J, Forrest AR, Suzuki H, Hayashizaki Y, Hume DA, Orlando V, Grimmond SM, Carninci P. The regulated retrotransposon transcriptome of mammalian cells. Nat Genet. 2009 May;41(5):563-71. doi: 10.1038/ng.368. Epub 2009 Apr 19. PMID: 19377475.

9. Djebali S, Davis CA, Merkel A, Dobin A, Lassmann T, Mortazavi A, Tanzer A, Lagarde J, Lin W, Schlesinger F, Xue C, Marinov GK, Khatun J, Williams BA, Zaleski C, Rozowsky J, Röder M, Kokocinski F, Abdelhamid RF, Alioto T, Antoshechkin I, Baer MT, Bar NS, Batut P, Bell K, Bell I, Chakrabortty S, Chen X, Chrast J, Curado J, Derrien T, Drenkow J, Dumais E, Dumais J, Duttagupta R, Falconnet E, Fastuca M, Fejes-Toth K, Ferreira P, Foissac S, Fullwood MJ, Gao H, Gonzalez D, Gordon A, Gunawardena H, Howald C, Jha S, Johnson R, Kapranov P, King B, Kingswood C, Luo OJ, Park E, Persaud K, Preall JB, Ribeca P, Risk B, Robyr D, Sammeth M, Schaffer L, See LH, Shahab A, Skancke J, Suzuki AM, Takahashi H, Tilgner H, Trout D, Walters N, Wang H, Wrobel J, Yu Y, Ruan X, Hayashizaki Y, Harrow J, Gerstein M, Hubbard T, Reymond A, Antonarakis SE, Hannon G, Giddings MC, Ruan Y, Wold B, Carninci P, Guigó R, Gingeras TR. Landscape of transcription in human cells. Nature. 2012 Sep 6;489(7414):101-8. doi: 10.1038/nature11233. PMID: 22955620; PMCID: PMC3684276.

10. Thurman RE, Rynes E, Humbert R, Vierstra J, Maurano MT, Haugen E, Sheffield NC, Stergachis AB, Wang $H$, Vernot B, Garg K, John S, Sandstrom R, Bates D, Boatman L, Canfield TK, Diegel M, Dunn D, Ebersol AK, Frum T, Giste E, Johnson AK, Johnson EM, Kutyavin T, Lajoie B, Lee BK, Lee K, London D, Lotakis D, Neph S, Neri F, Nguyen ED, Qu H, Reynolds AP, Roach V, Safi A, Sanchez ME, Sanyal A, Shafer A, Simon JM, Song L, Vong S, Weaver M, Yan Y, Zhang Z, Zhang Z, Lenhard B, Tewari M, Dorschner MO, Hansen RS, Navas PA, Stamatoyannopoulos G, lyer VR, Lieb JD, Sunyaev SR, Akey JM, Sabo PJ, Kaul R, Furey TS, Dekker J, Crawford GE, Stamatoyannopoulos JA. The accessible chromatin landscape of the human genome. Nature. 2012 Sep 6;489(7414):75-82. doi: 10.1038/nature11232. PMID: 22955617; PMCID: PMC3721348. 
Table 1: Comparison of RepeatMasker results between Set $\mathrm{T}$ and Set $\mathrm{N}$

\begin{tabular}{|c|c|c|}
\hline PARAMETERS/GENE SETS & Set $\mathbf{T}$ & Set $\mathbf{N}$ \\
\hline No. of genes & 1082 & 9454 \\
\hline GC level (\%) & 45.80 & 46.43 \\
\hline Bases masked (bp) & 42464.58 & 33748.54 \\
\hline Bases masked (\%) & 35.63 & 37.29 \\
\hline Number of SINEs & 66.37 & 47.33 \\
\hline Length of SINES (bp) & 15896.55 & 11190.46 \\
\hline Percentage of SINES & 16.99 & 16.73 \\
\hline Number of ALUs & 45.45 & 31.08 \\
\hline Length of ALUs (bp) & 12737.09 & 8717.72 \\
\hline Percentage of ALUs & 14.15 & 13.57 \\
\hline Number of MIRs & 20.52 & 16.01 \\
\hline Length of MIRs (bp) & 3107.57 & 2441.83 \\
\hline Percentage of MIRs & 2.80 & 3.14 \\
\hline Number of LINEs & 32.02 & 25.46 \\
\hline Length of LINEs (bp) & 15628.45 & 13881.68 \\
\hline Percentage of LINES & 10.52 & 11.66 \\
\hline Number of LINE1 & 16.19 & 12.90 \\
\hline Length of LINE1 (bp) & 11505.75 & 10429.40 \\
\hline Percentage of LINE1 & 7.10 & 7.96 \\
\hline Number of LINE2 & 13.31 & 10.62 \\
\hline Length of LINE2 (bp) & 3550.82 & 2992.54 \\
\hline Percentage of LINE2 & 2.99 & 3.29 \\
\hline Number of L3/CR1 & 1.92 & 1.46 \\
\hline Length of L3/CR1 (bp) & 429.94 & 334.75 \\
\hline Percentage of L3/CR1 & 0.32 & 0.32 \\
\hline Number of LTR & 10.15 & 8.93 \\
\hline Length of LTR (bp) & 4749.27 & 4270.76 \\
\hline Percentage of LTR & 2.75 & 3.67 \\
\hline Number of ERVL & 2.09 & 1.85 \\
\hline Length of ERVL (bp) & 990.63 & 882.81 \\
\hline Percentage of ERVL & 0.59 & 0.75 \\
\hline Number of ERVL-MaLRs & 5.46 & 4.74 \\
\hline Length of ERVL-MaLRs (bp) & 2241.70 & 1969.89 \\
\hline Percentage of ERVL-MaLRs & 1.27 & 1.49 \\
\hline Number of ERV_classI & 1.93 & 1.73 \\
\hline Length of ERV_classI (bp) & 1243.02 & 1172.28 \\
\hline Percentage of ERV_classI & 0.71 & 1.15 \\
\hline
\end{tabular}




\begin{tabular}{|c|c|c|}
\hline Number of ERV_classII & 0.14 & 0.12 \\
\hline Length of ERV_classII (bp) & 130.39 & 118.75 \\
\hline Percentage of ERV_classII & 0.10 & 0.16 \\
\hline Number of DNA_elements & 17.14 & 12.52 \\
\hline Length of DNA_elements (bp) & 4014.95 & 2938.19 \\
\hline Percentage of DNA_elements & 3.05 & 2.97 \\
\hline Number of hAT-Charlie (No.) & 9.22 & 6.68 \\
\hline Length of hAT-Charlie (bp) & 1885.93 & 1349.90 \\
\hline Percentage of hAT-Charlie & 1.48 & 1.45 \\
\hline Number of TcMar-Tigger & 4.33 & 3.08 \\
\hline Length of TcMar-Tigger (bp) & 1455.16 & 1063.87 \\
\hline Percentage of TcMar-Tigger & 1.13 & 0.98 \\
\hline Number of Unclassified & 2.78 & 1.85 \\
\hline Length of Unclassified (bp) & 359.91 & 245.65 \\
\hline Percentage of Unclassified & 0.36 & 0.36 \\
\hline Number of Total_interspersed_repeats & NA & NA \\
\hline Length of Total_interspersed_repeats (bp) & 40649.13 & 32526.74 \\
\hline Percentage of Total_interspersed_repeats & 33.67 & 35.39 \\
\hline Number of Small_RNA & 3.11 & 2.06 \\
\hline Length of Small_RNA & 202.00 & 133.31 \\
\hline Percentage of Small_RNA & 0.22 & 0.26 \\
\hline Number of Satellites & 0.04 & 0.02 \\
\hline Length of Satellites (bp) & 48.43 & 14.14 \\
\hline Percentage of Satellites & 0.02 & 0.08 \\
\hline Number of Simple_repeats & 27.75 & 19.11 \\
\hline Length of Simple_repeats (bp) & 1389.35 & 947.15 \\
\hline Percentage of Simple_repeats & 1.47 & 1.32 \\
\hline Number of Low_complexity & 3.06 & 2.20 \\
\hline Length of Low_complexity (bp) & 169.37 & 121.04 \\
\hline Percentage of Low_complexity & 0.23 & 0.23 \\
\hline
\end{tabular}


Table 2: Comparison of number of MGEs identified in Set $\mathrm{T}$ and different subsets of Set $\mathrm{N}$

\begin{tabular}{|c|c|c|c|c|c|c|}
\hline $\begin{array}{l}\text { PARAMETERS/GENE } \\
\text { SETS }\end{array}$ & Set $\mathbf{T}$ & Set N1 & Set $\mathbf{N 2}$ & Set N3 & Set N4 & Set N5 \\
\hline SINEs & 66.37 & 48.54 & 45.95 & 46.93 & 47.89 & 48.02 \\
\hline ALUs & 45.45 & 31.22 & 29.85 & 31.41 & 31.07 & 31.29 \\
\hline MIRs & 20.52 & 17.07 & 15.87 & 15.32 & 16.58 & 16.48 \\
\hline LINEs & 32.02 & 26.22 & 25.21 & 24.53 & 26.37 & 25.95 \\
\hline LINE1 & 16.19 & 13.13 & 12.74 & 12.49 & 13.24 & 13.07 \\
\hline LINE2 & 13.31 & 11.11 & 10.61 & 10.19 & 11.11 & 10.88 \\
\hline $\mathrm{L} 3 / \mathrm{CR} 1$ & 1.92 & 1.52 & 1.39 & 1.39 & 1.51 & 1.53 \\
\hline LTR & 10.15 & 9.06 & 9.27 & 8.40 & 9.28 & 9.04 \\
\hline ERVL & 2.09 & 1.87 & 1.96 & 1.79 & 1.94 & 1.92 \\
\hline ERVL-MaLRs & 5.46 & 4.82 & 4.92 & 4.40 & 4.87 & 4.78 \\
\hline ERV_classI & 1.93 & 1.78 & 1.79 & 1.70 & 1.79 & 1.75 \\
\hline ERV_classII & 0.14 & 0.12 & 0.12 & 0.11 & 0.14 & 0.12 \\
\hline DNA_elements & 17.14 & 12.94 & 12.26 & 11.75 & 12.69 & 13.09 \\
\hline hAT-Charlie & 9.22 & 6.86 & 6.53 & 6.22 & 6.66 & 7.04 \\
\hline TcMar-Tigger & 4.33 & 3.26 & 3.08 & 3.01 & 3.20 & 3.14 \\
\hline Unclassified & 2.78 & 1.88 & 1.80 & 1.92 & 1.83 & 1.86 \\
\hline
\end{tabular}




\begin{tabular}{|l|l|l|l|l|l|l|}
\hline Total_interspersed_repeats & NA & NA & NA & NA & NA & NA \\
\hline Small_RNA & 3.11 & 2.08 & 1.98 & 2.14 & 2.03 & 2.07 \\
\hline Satellites & 0.04 & 0.03 & 0.03 & 0.02 & 0.03 & 0.01 \\
\hline Simple_repeats & 27.75 & 19.54 & 18.94 & 18.39 & 19.50 & 19.31 \\
\hline Low_complexity & 3.06 & 2.22 & 2.20 & 2.02 & 2.29 & 2.27 \\
\hline
\end{tabular}




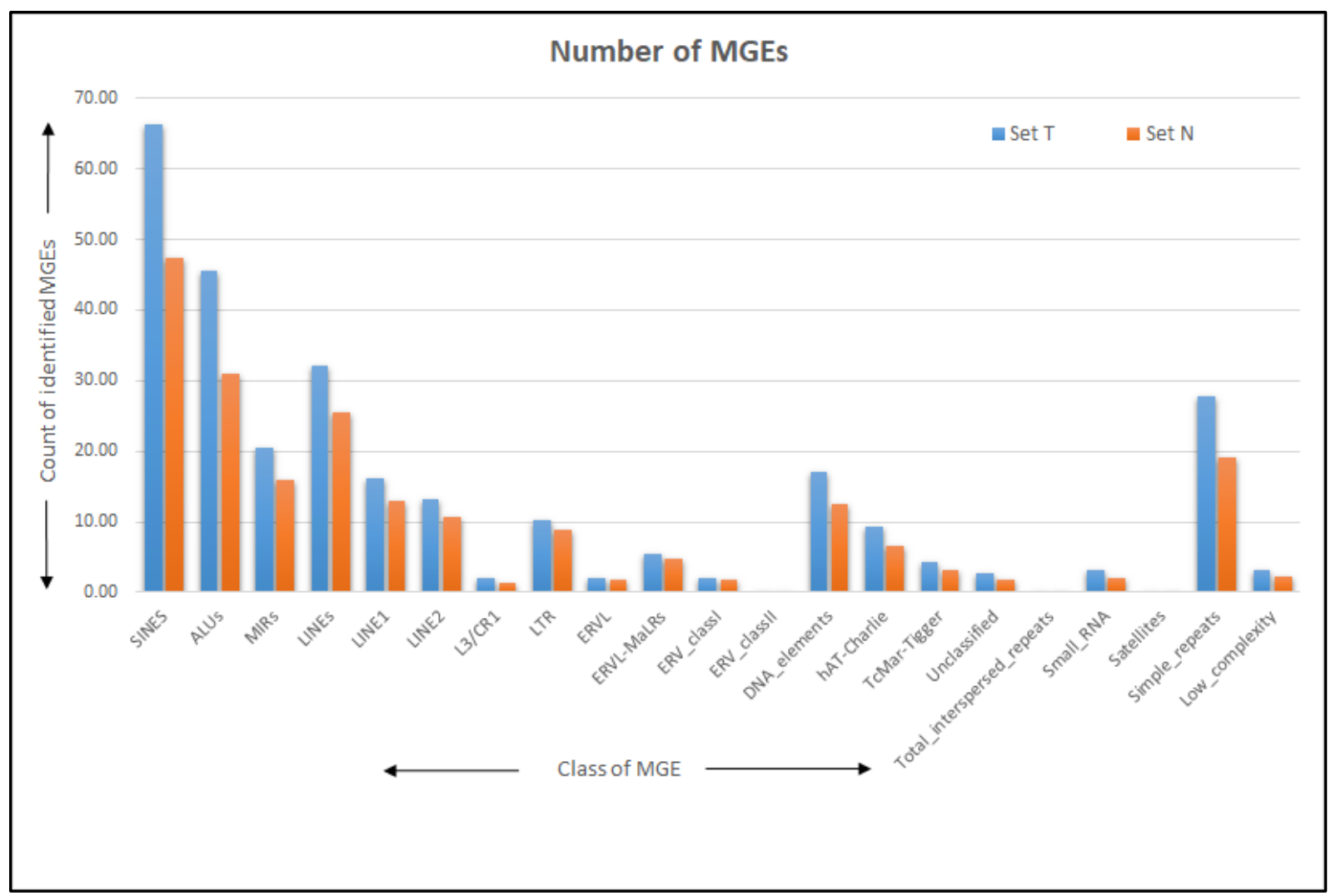

Figure 1. Comparison of number of MGEs between set $\mathrm{T}$ and set $\mathrm{N}$. The $y$-axis represents the number of MGEs identified and the $x$ - axis represents the different class of MGEs. 


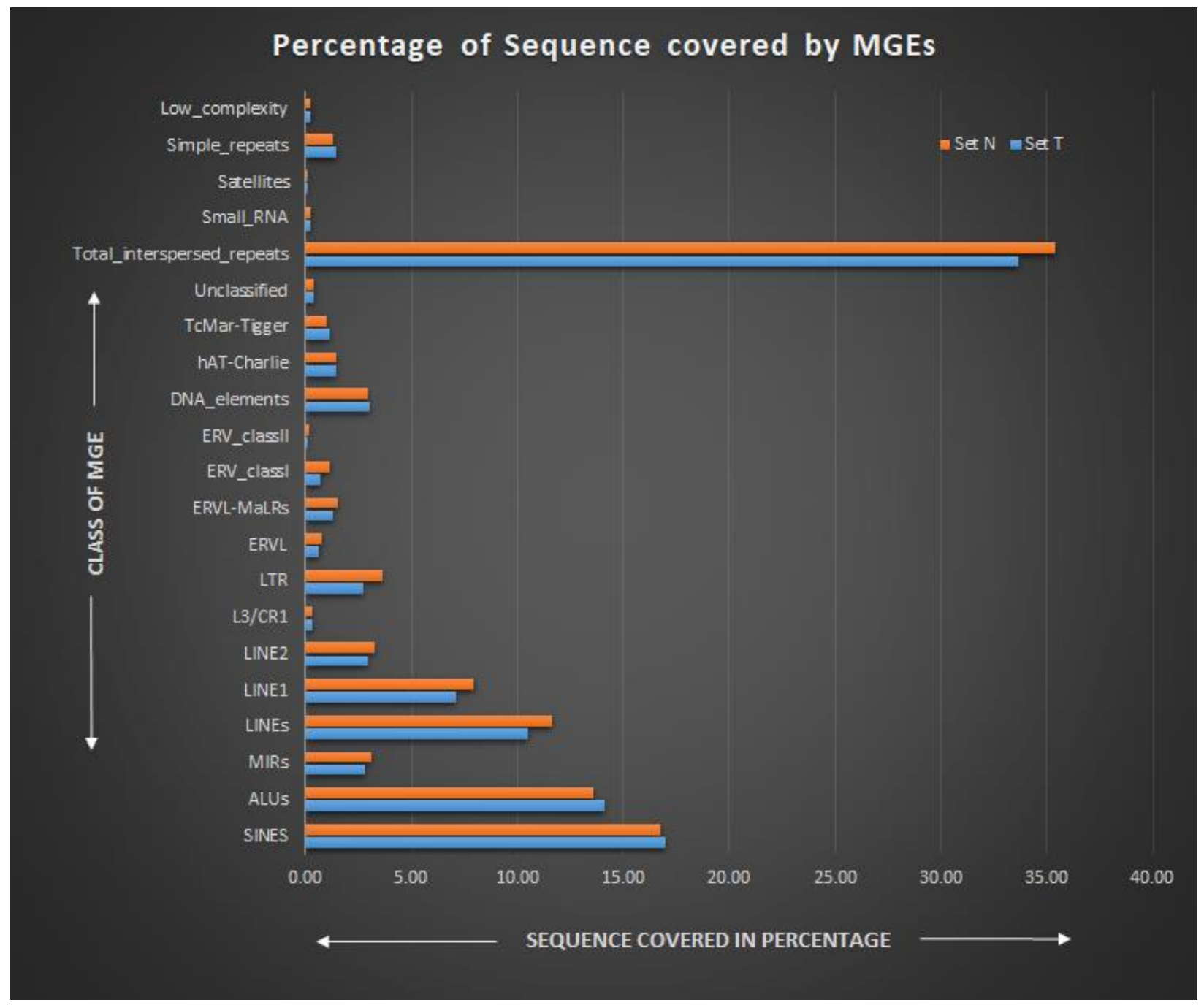

Figure 2. Comparison of percentage of sequence covered by MGEs between set $\mathrm{T}$ and set $\mathrm{N}$. The $y$-axis represents the different class of MGEs and the $x$-axis represents the sequence covered by each class of MGE (in percentage). 


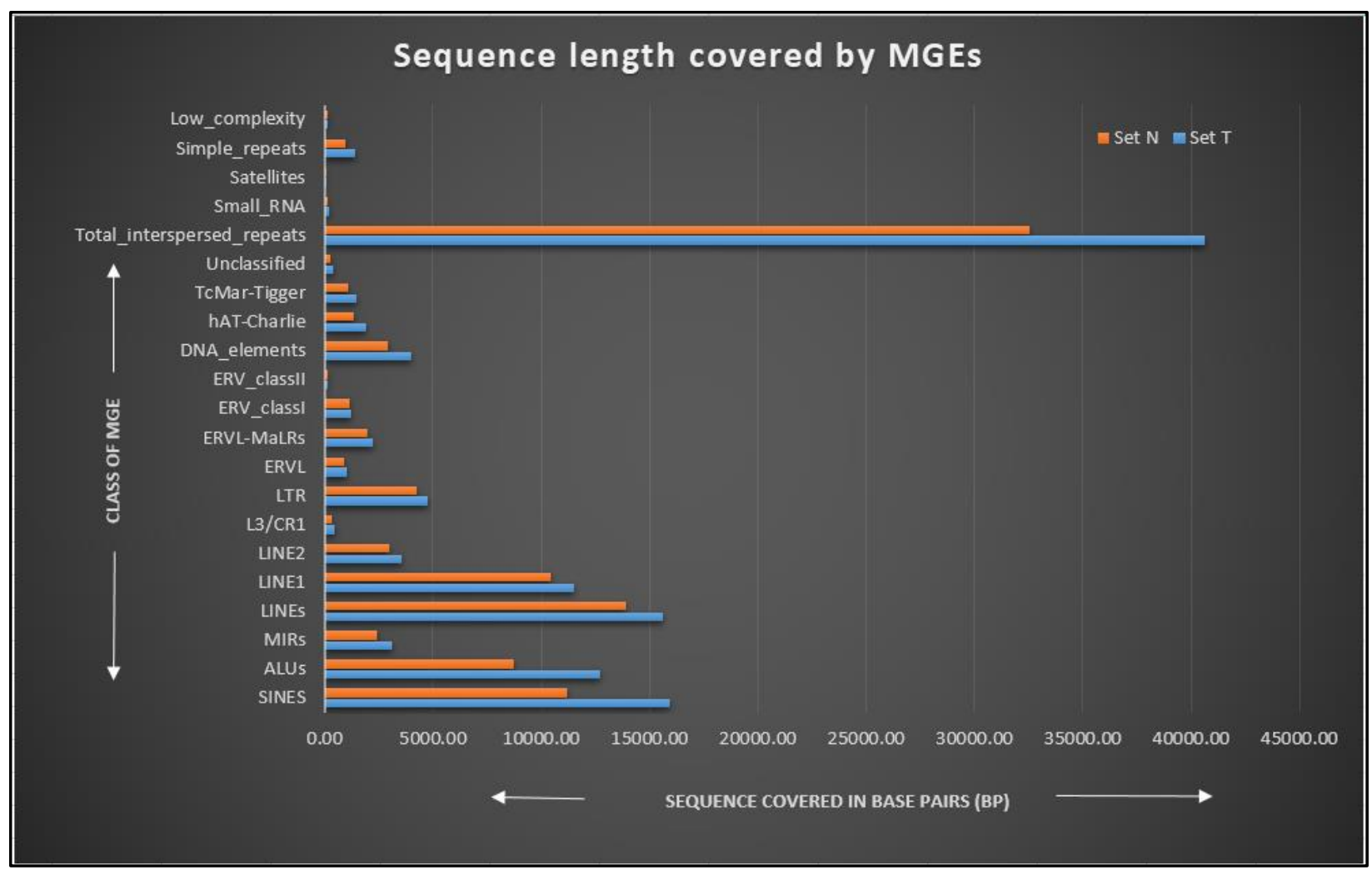

Figure 3. Comparison of sequence length covered by MGEs between set T and set $\mathrm{N}$. The $y$ - axis represents the different class of MGEs and the $x$ - axis represents the sequence covered (in base pairs) by each class of MGE. 